\title{
Polyphosphate as a haemostatic modulator
}

Nicola .J. Mutch

Institute of Medical Sciences, University of Aberdeen, Aberdeen, UK

\section{Correspondence:}

Dr Nicola J Mutch

Institute of Medical Sciences

University of Aberdeen

Aberdeen

AB25 2ZD

UK

Tel: 441224437492

n.j.mutch@abdn.ac.uk

Running title: Polyphosphate \& haemostasis

Text: 2751 words

Synopsis: 225 words

Keywords: Polyphosphate, Coagulation, Fibrinolysis, Haemostasis, Platelets, Fibrin 


\section{Synopsis}

Platelets are small anuclear cells that play a central role in haemostasis. Platelets become activated in response to various stimuli triggering release of their granular contents into the surrounding milieu. One of these types of granules, termed dense granules, have been found to contain polyP in addition to other inorganic biomolecules, such as serotonin, ADP, ATP, PPi. Individuals deficient in dense granules exhibit bleeding tendencies, emphasising their importance in haemostasis. Platelet polyP is of a relatively defined size, approximately 60-100 phosphate monomers in length. These linear polymers act at various points in the coagulation and fibrinolytic systems thereby modulating the haemostatic response. Due to its highly anionic nature polyP lends itself to being a natural activator of the contact system. The contact system functions in multiple pathways including coagulation, fibrinolysis, inflammation and complement. Activation of the contact system accelerates thrombin generation, the terminal enzyme in the coagulation cascade. PolyP also modulates factors further downstream in the coagulation cascade to augment thrombin generation. The net effect is increased fibrin formation and platelet activation resulting in faster clot formation. PolyP is incorporated into the forming clot thereby modifying the structure of the resulting fibrin network and its susceptibility to degradation by certain plasminogen activators. In conclusion, release of platelet polyP at the site of injury may facilitate clot formation and augment clot stability thereby promoting wound healing. 
Polyphosphate (polyP) is a ubiquitous molecule present in all living organisms and is highly conserved throughout evolution [1]. It is a linear unbranched polymer of inorganic phosphate residues linked by energy-rich phosphoanhydride bonds, such as those found in ADP, which varies considerably in length depending on the organism and tissue of synthesis. The majority of our knowledge of polyP synthesis and regulation is derived from prokaroyotes and unicellular eurokaryotes, where a $\gamma$-phosphate of ATP is transferred to a growing chain of phosphate residues by an enzymatic reaction [1]. PolyP can be degraded through the action of exophosphatases and endophosphatases, which remove phosphate resides from either termini or cleave internal phosphoanhydride bonds, respectively. The potential of polyP to function in metabolic processes is underscored by its chemical and physical properties, such as its strong anionic nature, its ability to form high energy bonds and its capacity to complex with $\mathrm{Ca}^{2+}$ and other divalent cations. There is a wealth of literature on the functions of polyP in prokaryotes and single-cell eukaryotes [2] where it essential for normal physiological processes such as motility, virulence and stress responses [3]. However, until recently the role of this polymer in mammalian systems was relatively undefined. In 2004, Ruiz et al [4] established distinct similarities between acidocalcisomes in bacteria and the dense granules contained within human platelets, as both types of organelles are acidic in nature [5], electron dense, contain high concentrations of divalent cations and are a rich source of ADP/ATP as well as inorganic phosphate [6]. Further purification of these dense granules (sometimes referred to as delta granules) revealed that they also store high concentrations of polyP. This review will focus on the haemostatic functions of platelet-derived polyP that have been uncovered in the last decade since its identification in these small anucleate blood cells.

\section{Platelets and polyP}

Platelets are the smallest cell in blood with a diameter of 2-5 $\mu \mathrm{m}$ and circulate at high concentrations $\left(\sim 1.5-4.5 \times 10^{8} / \mathrm{ml}\right)$. Platelets are crucial to the haemostatic response and provide the first point of contact with the damaged endothelium by interacting with collagen and von Willebrand factor, thereby forming an initial platelet plug (an event termed primary haemostasis). Their role in haemostasis does not end here as they continue to support secondary haemostasis by providing a procoagulant surface for assembly of plasma derived coagulation factors and ultimately facilitate generation of the clotting enzyme thrombin. They also provide an anchor for fibrin, via the integrin receptor $\alpha_{11 b} \beta_{3}$, to support the growing clot and release a host of proteins from their $\alpha$ granules and modulators from their dense granules to regulate clot formation, stability and degradation.

PolyP is contained at high concentration of around $130 \mathrm{mM}$ within platelet dense granules and is of a narrow size range, compared to long-chain microbial polyP, of around 60-100 phosphate monomers in length. The pool of polyP is released into the secretome of platelets following activation by such as thrombin, ADP and collagen, alongside other dense granule contents including ADP and serotonin $[4,7]$. Secretion of polyP upon platelet stimulation results in release of low micromolar concentrations of polyP into the circulation (expressed in terms of phosphate monomer). Once released polyP is likely to have a relatively limited half-life of $1-2 \mathrm{~h}$ in plasma before it is degraded by phosphatase enzymes [8].

The crucial role of platelets in the haemostatic response and the fact that individuals with defects in dense granules exhibit bleeding tendencies [9] prompted our analysis of the functional effects of polyP in these processes. Using synthetic polyP, of approximately the size found in platelets, we demonstrated that this polymer can significantly augment coagulation and delay fibrinolysis (Figure 1A) [8]. PolyP-mediated acceleration of coagulation occurs at several points in the cascade including initiating activation of the contact pathway (Figure 1B) [8], accelerating thrombinmediated activation of factor $\mathrm{XI}[10]$ and enhancing conversion of the procofactor factor $\mathrm{V}$ to factor 
Va [8]. The effect of polyP on thrombin generation has down-stream repercussions in terms of fibrinolysis, where it augments activation of the fibrinolytic modulator, thrombin activatable fibrinolysis inhibitor (TAFI). PolyP also binds to a number of the haemostatic proteins including thrombin [11], kallikrein [12], factor XI (FXI) [10] and factor XII (FXII) [7, 13]. It also associates with fibrinogen [14] which, once cleaved by thrombin to fibrin, provides the main scaffold of the forming clot. These observations led to the hypothesis that polyP operates as a timed switch, to enhance repair of an injured region while simultaneously preventing clot breakdown until wound healing occurs [8]. These multifaceted effects of polyP on haemostasis will now be discussed in more detail.

\section{PolyP-mediated effects on the contact pathway}

The contact pathway is composed of four proteins, factor XII (FXII), prekallikrein (PK), factor XI (FXI) and high molecular weight kininogen (HK) (Figure 2). Reciprocal proteolytic activation of FXII and PK to their active forms, FXIla and kallikrein respectively, is stimulated by negatively charged surfaces (Figure 2). FXIla then cleaves FXI to its activated form, FXIa. FXI and PK circulate in complex with the non-enzymatic cofactor $\mathrm{HK}$, which assembles these proteases onto the activating surface. Zinc ions induce a conformational change in FXII [15-19] and HK [20-22], enhancing surface interactions. The function of FXII in coagulation has been questioned as congenital deficiencies are not associated with bleeding in humans [23] or in mice [24, 25]. Advances in defining the pathophysiological importance of the contact pathway have been made using FXII-deficient mice, which show normal haemostasis and early thrombus formation but a clear defect in thrombus stabilisation [26, 27]. A major obstacle in defining the physiological role of this pathway has been the lack of an appropriate biological surface capable of initiating contact activation. However, in the past decade our work [7, 8,13 ] and others [28-30] has identified natural surfaces such as, RNA [28], misfolded proteins [29], collagen [30] and, relevant to this review, polyP $[7,8,13]$, that all fuel FXII activation.

FXII has several putative binding sites for anionic surfaces within the fibronectin type II [31-33] and type I domains [34]. Cleavage at Arg353-Val354 generates $\alpha$ FXIla, a disulphide linked two-chain protein composed of a heavy chain and light chain. Further cleavage at Arg334-Val335 generates $\beta F X I l a$, a proteolytic form that lacks the surface binding domains. BFXIla can cleave PK at similar rates to $\alpha \mathrm{FXIla}$ but is an inefficient activator of FXI [35]. PolyP binds directly to FXII and can induce autoactivation and activation by kallikrein [7], although the exact location of this binding site has not been elucidated. Interestingly, we have shown that polyP, of around the chain length in platelets, when bound to FXII can induce activity in the single-chain form of the protease [13]. This FXII-polyP complex is functional in cleaving downstream targets of FXII including FXI and kallikiren and is efficiently inhibited by the physiological inhibitor $\mathrm{C} 1$-inhibitor. In contrast, longer chain polyP ( $\geq 500$ mer) are much more efficient at stimulating FXII activation [36] and drive formation of the cleaved two chain $\alpha F X I l a$ form. These data are consistent with previous observations showing that autoactivation of FXII is induced more slowly by low molecular mass polysaccharides, than higher molecular mass polysaccharides of $10000 \mathrm{Da}$ and above [31]. The increased level of autoactivation observed with these different chain lengths of polymers is rationalised by the increase in available binding sites on longer polymers. Assuming a bond length of $\mathrm{P}-\mathrm{O}$ as $1.5 \AA$, we can derive the length of polyP 70 as approximately $20 \mathrm{~nm}$. The diameter of FXII is $5-6 \mathrm{~nm}(\mathrm{Mr}=80000)$ based on the assumption that the protein molecule is approximately spherical [31]. These approximate calculations infer that around four FXII molecules could bind to polyP $\mathrm{P}_{70}$ which may account for the levels of autoactivation and activity observed [13]. These data provide novel insights into the subtleties that regulate FXII autoactivation by naturally occurring anionic surfaces and suggest that when FXII is in association with different 'cofactor surfaces' it may have different functional activity.

Platelet-derived polyP has been identified as an efficient stimulator of autoactivation and thrombinmediated activation of FXI [10]. PolyP is known to bind to thrombin via exosite II [10, 11] and factor 
$\mathrm{XI}$ via the anion binding sites $[7,10,37]$ therefore it is likely that polyP elicits it functions via a template mechanism. In this model proteases bind to the surface via distinct exosites thereby lowering the dissociation constant and enhancing interaction with their target zymogens. This is a familiar mechanism in haemostasis in terms of heparin-enhanced inhibition of thrombin by antithrombin [38] and fibrin-stimulated activation of plasminogen by tissue plasminogen activator (tPA) [39]. The study by Choi et al [10] suggests that within the plasma milieu the presence of both polyP and thrombin could potentiate thrombin generation via autoactivation of FXI or enhanced thrombin-mediated cleavage of FXI. Interestingly, binding of polyP to FXI also accelerates downstream factor $V$ activation, acting at a further point to accelerate local thrombin generation [40]. These observations could help to explain the mild bleeding phenotype associated with FXI deficiency (Haemophilia C), which is absent in FXII deficient individuals.

Since the discovery that the contact pathway is dispensable for haemostasis but functions in thrombosis it has been pinpointed as a safe target for antithrombotic drugs that can inhibit clot formation with minimal bleeding complications [41]. A recent study has also elegantly demonstrated that antisense inhibition of FXI decreased the degree of deep vein thrombosis after knee arthroplasty, defining a key role for FXla in mediating thrombus formation in vivo [42]. The importance of the contact pathway in fibrin deposition has recently been reported in a flow-based model using human blood [43]. The authors show that inhibition of FXIla-mediated and thrombinmediated FXI activation reduces fibrin formation while having no direct impact on platelet deposition [43]. Interestingly, inclusion of a polyP binding protein modulated fibrin deposition at low TF concentrations suggesting a contact system-independent effect on the extrinsic pathway [43]. In collaboration with the Renne laboratory and others [7] we have shown that intravenous administration of polyP into mice results in fatal pulmonary embolism, however mice deficient in FXII or wild-type mice treated with a FXII inhibitor survive this challenge, illustrating a clear role for polyP in modulating FXII activation in vivo [7]. Recent observations have shown the polyP-FXII pathway drives thrombosis in prostate cancer [44]. Prostate cancer cells and the exosome they release (prostasomes) expose long-chain polyP on their surface that triggers FXII activation and downstream clotting in prostate cancer patient plasma and thrombosis in mice [44]. Inhibition of the polyP-FXII pathway reduces thrombosis in mice with no bleeding complications identifying this pathway as a novel therapeutic target for anticoagulant drug development for the treatment of prostate cancer-induced thrombosis. Indeed, studies are already underway to identify polyP inhibitors and have been shown to reduce venous and arterial thrombosis in vivo [45]. Travers et al [46] demonstrate that non-toxic dendritic polymer based compounds were efficient at modulating polyP induced coagulation in vitro and in vivo. This highlights the possibly of future drugs based on similar technology that could be used to treat various types of thromboembolic complications.

\section{PolyP enhances thrombin generation}

PolyP significantly augments thrombin generation [8] suggesting it has a direct effect on the common pathway (Figure 2). Factor $\mathrm{V}$ is the circulating protein procofactor for factor $\mathrm{Va}$, which is essential for prothrombin activation by factor Xa. Our initial studies into polyP's function in coagulation revealed that it accelerates conversion of factor $\mathrm{V}$ to factor $\mathrm{Va}$ by both factor $\mathrm{Xa}$ and thrombin [8], of which the latter is considered to be the principal activator in vivo [47]. Enhanced activation of factor $V$ occurs readily with polyP of the size found in platelets [36]. Platelet $\alpha$-granules store a proteolytically modified form of factor Va which is more susceptible to activation $[48,49]$. Activated platelets provide a surface for binding of the coagulation factors, specifically factor $X$, prothrombin and factor $V$. Taken together this suggests that concomitant release of platelet polyP and platelet factor $\mathrm{V}$ is an important route for augmenting local thrombin generation on the surface of activated platelets. 
The amplified conversion of factor $\mathrm{V}$ to factor $\mathrm{Va}$ in the presence of polyP has intriguing downstream consequences in terms of regulation of coagulation. TFPI is the primary inhibitor of tissue factor driven coagulation, acting at several key points to regulate the pathway. One of the most important points of interception is inhibition of factor Xa before it dissociates from the TF-FVIla complex [50]. Interestingly, factor $\mathrm{Xa}$ is protected from inhibition by TFPI when in complex with its cofactor factor $\mathrm{Va}$, particularly in the presence of prothrombin [51]. We found that addition of platelet releasates attenuated TFPI function in a polyP-dependent manner [8]. Therefore not only does polyP accelerate formation of activated clotting factors it also interferes with the ability of endogenous inhibitors to modulate coagulation.

\section{PolyP modulates fibrin formation and stability}

Thrombin is responsible for cleaving circulating fibrinogen to fibrin and is actively incorporated into the growing fibrin network. Thrombin also activates factor XIII, a transglutaminase enzyme whose function is to cross-link fibrin and inhibitors, specifically $\alpha_{2} A P$, into thrombi to stabilise them against mechanical [52,53] and fibrinolytic degradation [54], respectively. PolyP is known to bind to fibrin [14] and is incorporated into the clot modifying the structure of the resulting fibrin network $[14,55]$. We observed that fibrin formed in the presence of polyP was extremely heterogeneous in nature, being composed of small knotted regions interspersed by large pores (Figure 3) [14]. Intriguingly, polyP is located in the centre of these knotted areas (unpublished observations Whyte CS \& Mutch NJ), as if it functions as a nucleus for fibrin formation. Fibrinolysis is the enzymatic process by which fibrin is degraded. Plasmin, the central enzyme in fibrinolysis, is formed by activation of the zymogen plasminogen by a plasminogen activator, namely tissue plasminogen activator (tPA) and urokinase (UPA) (Figure 2). Both tPA and plasminogen bind avidly to C-terminal lysine residues on the fibrin surface and intriguingly fibrin acts as a cofactor in its own destruction. A key consequence of the altered fibrin structure induced by polyP is attenuation of tPA-mediated fibrinolysis. This is in part mediated by the modified fibrin network, but perhaps more importantly because polyP tempers the binding of the fibrinolytic proteins plasminogen and tPA to fibrin (Figure 3B). The exact mechanism is not yet defined, but suggest that deficient binding arises from obstruction of the C-terminal lysine residues, particularly as these effects are more pronounced following plasmin hydrolysis of fibrin which exposes further C-terminal lysine binding sites (Figure 3B) [14].

TAFI is a procarboxypeptidase that is activated to the enzyme TAFla via thrombin [56] and plasmin [57]. TAFla modulates fibrinolysis by cleaving the C-terminal lysine residues from fibrin attenuating binding of plasminogen and tPA and thereby reducing plasmin generation. By enhancing thrombin generation via the multiple mechanisms described $[7,8,10,40]$ polyP increases thrombinmediated TAFI activation and subsequently down-regulates fibrinolysis (Figure 2 ). These observations indicate that incorporation of polyP into forming clots stabilises the fibrin network by different mechanisms to prevent premature degradation of the clot but in pathological situations this may allow clots to persist within the vasculature.

\section{Conclusions and future perspectives}

The work discussed in this review illustrates that platelet polyP modulates haemostasis at several distinct points in the cascade. It is fascinating that a simple molecule like polyP has the capacity to mediate these crucial physiological processes. Several of the procoagulant effects of polyP are mediated by its anionic charge and are consistent with a template mechanism $[10,11,36]$ and/or allosteric modulation of proteins $[11,13]$. However, many of these mechanisms are still relatively undetermined and will undoubtedly prove to be an area of important research in future years. While polyP of the size found in platelets is a fairly modest activator of FXII the fact that both FXII [58] and polyP [14, 55] are localised on fibrin suggests that it may have site specific pathobiological importance. The role of polyP is 
particularly relevant when considering bacterial induced infection, such as sepsis, as bacteria contain high concentrations of long-chain polyP, which are potent stimulators of FXII activation [36]. It is interesting to speculate that compounds that target polyP may provide important new strategies for treating thrombotic complications without the bleeding side-effects that are associated with current antithrombotic drugs. The evolutionary conservation of polyP emphasises its biological importance throughout the animal kingdom. The growing body of reports, however, accentuate the current lack of knowledge on its synthesis and regulation in mammalian cells and underscores a requirement to identify the enzymes responsible for its synthesis and metabolism in vivo.

\section{ACKNOWLEDGEMENTS}

With thanks to the British Heart Foundation for financial support (FS/11/2/28579).

\section{DISCLOSURE OF CONFLICT OF INTEREST}

The author has no conflicts of interest to disclose. 
References

1. Kornberg, A., Rao, N. N. \& Ault-Riche, D. (1999) Inorganic polyphosphate: a molecule of many functions, Annu Rev Biochem. 68, 89-125.

2. Kornberg, A. (1999) Inorganic polyphosphate: a molecule of many functions, Prog Mol Subcell Biol. 23, 1-18.

3. Brown, M. R. \& Kornberg, A. (2004) Inorganic polyphosphate in the origin and survival of species, Proc Natl Acad Sci U S A. 101, 16085-7.

4. Ruiz, F. A., Lea, C. R., Oldfield, E. \& Docampo, R. (2004) Human platelet dense granules contain polyphosphate and are similar to acidocalcisomes of bacteria and unicellular eukaryotes, J Biol Chem. 279, 44250-7.

5. White, J. G. (1969) The dense bodies of human platelets: inherent electron opacity of the serotonin storage particles, Blood. 33, 598-606.

6. Fukami, M. H., Dangelmaier, C. A., Bauer, J. S. \& Holmsen, H. (1980) Secretion, subcellular localization and metabolic status of inorganic pyrophosphate in human platelets. A major constituent of the amine-storing granules, Biochem J. 192, 99-105.

7. Muller, F., Mutch, N. J., Schenk, W. A., Smith, S. A., Esterl, L., Spronk, H. M., Schmidbauer, S., Gahl, W. A., Morrissey, J. H. \& Renne, T. (2009) Platelet polyphosphates are proinflammatory and procoagulant mediators in vivo, Cell. 139, 1143-56.

8. Smith, S. A., Mutch, N. J., Baskar, D., Rohloff, P., Docampo, R. \& Morrissey, J. H. (2006) Polyphosphate modulates blood coagulation and fibrinolysis, Proc Natl Acad Sci U S A. 103, 903-8.

9. Hernandez-Ruiz, L., Saez-Benito, A., Pujol-Moix, N., Rodriguez-Martorell, J. \& Ruiz, F. A. (2009) Platelet inorganic polyphosphate decreases in patients with delta storage pool disease, $J$ Thromb Haemost. 7, 361-3.

10. Choi, S. H., Smith, S. A. \& Morrissey, J. H. (2011) Polyphosphate is a cofactor for the activation of factor XI by thrombin, Blood. 118, 6963-70.

11. Mutch, N. J., Myles, T., Leung, L. L. \& Morrissey, J. H. (2010) Polyphosphate binds with high affinity to exosite II of thrombin, J Thromb Haemost. 8, 548-55.

12. Choi, S. H., Collins, J. N., Smith, S. A., Davis-Harrison, R. L., Rienstra, C. M. \& Morrissey, J. H. (2010) Phosphoramidate end labeling of inorganic polyphosphates: facile manipulation of polyphosphate for investigating and modulating its biological activities, Biochemistry. 49, 9935-41. 13. Engel, R., Brain, C. M., Paget, J., Lionikiene, A. S. \& Mutch, N. J. (2014) Single-chain factor XII exhibits activity when complexed to polyphosphate, J Thromb Haemost. 12, 1513-22. 
14. Mutch, N. J., Engel, R., Uitte de Willige, S., Philippou, H. \& Ariens, R. A. (2010) Polyphosphate modifies the fibrin network and down-regulates fibrinolysis by attenuating binding of tPA and plasminogen to fibrin, Blood. 115, 3980-8.

15. Shore, J. D., Day, D. E., Bock, P. E. \& Olson, S. T. (1987) Acceleration of surface-dependent autocatalytic activation of blood coagulation factor XII by divalent metal ions, Biochemistry. 26, 2250-8.

16. Schousboe, I. (1990) The inositol-phospholipid-accelerated activation of prekallikrein by activated factor XII at physiological ionic strength requires zinc ions and high-Mr kininogen, Eur J Biochem. 193, 495-9.

17. Bernardo, M. M., Day, D. E., Halvorson, H. R., Olson, S. T. \& Shore, J. D. (1993) Surfaceindependent acceleration of factor XII activation by zinc ions. II. Direct binding and fluorescence studies, J Biol Chem. 268, 12477-83.

18. Bernardo, M. M., Day, D. E., Olson, S. T. \& Shore, J. D. (1993) Surface-independent acceleration of factor XII activation by zinc ions. I. Kinetic characterization of the metal ion rate enhancement, $J$ Biol Chem. 268, 12468-76.

19. Shimada, T., Kato, H. \& Iwanaga, S. (1987) Accelerating effect of zinc ions on the surfacemediated activation of factor XII and prekallikrein, J Biochem (Tokyo). 102, 913-21.

20. Greengard, J. S. \& Griffin, J. H. (1984) Receptors for high molecular weight kininogen on stimulated washed human platelets, Biochemistry. 23, 6863-9.

21. Zini, J. M., Schmaier, A. H. \& Cines, D. B. (1993) Bradykinin regulates the expression of kininogen binding sites on endothelial cells, Blood. 81, 2936-46.

22. Herwald, H., Morgelin, M., Svensson, H. G. \& Sjobring, U. (2001) Zinc-dependent conformational changes in domain D5 of high molecular mass kininogen modulate contact activation, Eur J Biochem. 268, 396-404.

23. Ratnoff, O. D. \& Colopy, J. E. (1955) A familial hemorrhagic trait associated with a deficiency of a clot-promoting fraction of plasma, J Clin Invest. 34, 602-13.

24. Gailani, D., Lasky, N. M. \& Broze, G. J., Jr. (1997) A murine model of factor XI deficiency, Blood Coagul Fibrinolysis. 8, 134-44.

25. Pauer, H. U., Renne, T., Hemmerlein, B., Legler, T., Fritzlar, S., Adham, I., Muller-Esterl, W., Emons, G., Sancken, U., Engel, W. \& Burfeind, P. (2004) Targeted deletion of murine coagulation factor XII gene-a model for contact phase activation in vivo, Thromb Haemost. 92, 503-8.

26. Renne, T., Pozgajova, M., Gruner, S., Schuh, K., Pauer, H. U., Burfeind, P., Gailani, D. \& Nieswandt, B. (2005) Defective thrombus formation in mice lacking coagulation factor XII, J Exp Med. $202,271-81$. 
27. Kleinschnitz, C., Stoll, G., Bendszus, M., Schuh, K., Pauer, H. U., Burfeind, P., Renne, C., Gailani, D., Nieswandt, B. \& Renne, T. (2006) Targeting coagulation factor XII provides protection from pathological thrombosis in cerebral ischemia without interfering with hemostasis, J Exp Med. 203, 513-8.

28. Kannemeier, C., Shibamiya, A., Nakazawa, F., Trusheim, H., Ruppert, C., Markart, P., Song, Y., Tzima, E., Kennerknecht, E., Niepmann, M., von Bruehl, M. L., Sedding, D., Massberg, S., Gunther, A., Engelmann, B. \& Preissner, K. T. (2007) Extracellular RNA constitutes a natural procoagulant cofactor in blood coagulation, Proc Natl Acad Sci U S A. 104, 6388-93.

29. Maas, C., Govers-Riemslag, J. W., Bouma, B., Schiks, B., Hazenberg, B. P., Lokhorst, H. M., Hammarstrom, P., ten Cate, H., de Groot, P. G., Bouma, B. N. \& Gebbink, M. F. (2008) Misfolded proteins activate factor XII in humans, leading to kallikrein formation without initiating coagulation, $J$ Clin Invest. 118, 3208-18.

30. van der Meijden, P. E., Munnix, I. C., Auger, J. M., Govers-Riemslag, J. W., Cosemans, J. M., Kuijpers, M. J., Spronk, H. M., Watson, S. P., Renne, T. \& Heemskerk, J. W. (2009) Dual role of collagen in factor XII-dependent thrombus formation, Blood. 114, 881-90.

31. Clarke, B. J., Cote, H. C., Cool, D. E., Clark-Lewis, I., Saito, H., Pixley, R. A., Colman, R. W. \& MacGillivray, R. T. (1989) Mapping of a putative surface-binding site of human coagulation factor XII, J Biol Chem. 264, 11497-502.

32. Samuel, M., Samuel, E. \& Villanueva, G. B. (1993) Histidine residues are essential for the surface binding and autoactivation of human coagulation factor XII, Biochem Biophys Res Commun. 191, 110-7.

33. Pixley, R. A., Stumpo, L. G., Birkmeyer, K., Silver, L. \& Colman, R. W. (1987) A monoclonal antibody recognizing an icosapeptide sequence in the heavy chain of human factor XII inhibits surface-catalyzed activation, J Biol Chem. 262, 10140-5.

34. Citarella, F., Ravon, D. M., Pascucci, B., Felici, A., Fantoni, A. \& Hack, C. E. (1996)

Structure/function analysis of human factor XII using recombinant deletion mutants. Evidence for an additional region involved in the binding to negatively charged surfaces, Eur J Biochem. 238, 240-9. 35. Revak, S. D., Cochrane, C. G., Bouma, B. N. \& Griffin, J. H. (1978) Surface and fluid phase activities of two forms of activated Hageman factor produced during contact activation of plasma, $J$ Exp Med. 147, 719-29.

36. Smith, S. A., Choi, S. H., Davis-Harrison, R., Huyck, J., Boettcher, J., Rienstra, C. M. \& Morrissey, J. H. (2010) Polyphosphate exerts differential effects on blood clotting, depending on polymer size, Blood. 116, 4353-9. 
37. Geng, Y., Verhamme, I. M., Smith, S. A., Cheng, Q., Sun, M., Sheehan, J. P., Morrissey, J. H. \& Gailani, D. (2013) Factor XI anion-binding sites are required for productive interactions with polyphosphate, J Thromb Haemost. 11, 2020-8.

38. Li, W., Johnson, D. J., Esmon, C. T. \& Huntington, J. A. (2004) Structure of the antithrombinthrombin-heparin ternary complex reveals the antithrombotic mechanism of heparin, Nat Struct Mol Biol. 11, 857-62.

39. Horrevoets, A. J., Pannekoek, H. \& Nesheim, M. E. (1997) A steady-state template model that describes the kinetics of fibrin-stimulated [Glu1]- and [Lys78]plasminogen activation by native tissuetype plasminogen activator and variants that lack either the finger or kringle-2 domain, J Biol Chem. 272, 2183-91.

40. Choi, S. H., Smith, S. A. \& Morrissey, J. H. (2015) Polyphosphate accelerates factor V activation by factor Xla, Thromb Haemost. 113, 599-604.

41. Kenne, E. \& Renne, T. (2014) Factor XII: a drug target for safe interference with thrombosis and inflammation, Drug Discov Today. 19, 1459-64.

42. Buller, H. R., Bethune, C., Bhanot, S., Gailani, D., Monia, B. P., Raskob, G. E., Segers, A., Verhamme, P., Weitz, J. I. \& Investigators, F.-A. T. (2015) Factor XI antisense oligonucleotide for prevention of venous thrombosis, N Engl J Med. 372, 232-40.

43. Zhu, S., Travers, R. J., Morrissey, J. H. \& Diamond, S. L. (2015) FXla and platelet polyphosphate as therapeutic targets during human blood clotting on collagen/tissue factor surfaces under flow, Blood.

44. Nickel, K. F., Ronquist, G., Langer, F., Labberton, L., Fuchs, T. A., Bokemeyer, C., Sauter, G., Graefen, M., Mackman, N., Stavrou, E. X., Ronquist, G. \& Renne, T. (2015) The polyphosphate-factor XII pathway drives coagulation in prostate cancer-associated thrombosis, Blood. 126, 1379-89.

45. Smith, S. A., Choi, S. H., Collins, J. N., Travers, R. J., Cooley, B. C. \& Morrissey, J. H. (2012) Inhibition of polyphosphate as a novel strategy for preventing thrombosis and inflammation, Blood. 120, 5103-10.

46. Travers, R. J., Shenoi, R. A., Kalathottukaren, M. T., Kizhakkedathu, J. N. \& Morrissey, J. H. (2014) Nontoxic polyphosphate inhibitors reduce thrombosis while sparing hemostasis, Blood. 124, 318390.

47. Orfeo, T., Brufatto, N., Nesheim, M. E., Xu, H., Butenas, S. \& Mann, K. G. (2004) The factor V activation paradox, J Biol Chem. 279, 19580-91.

48. Monkovic, D. D. \& Tracy, P. B. (1990) Activation of human factor V by factor Xa and thrombin, Biochemistry. 29, 1118-28. 
49. Gould, W. R., Silveira, J. R. \& Tracy, P. B. (2004) Unique in vivo modifications of coagulation factor $V$ produce a physically and functionally distinct platelet-derived cofactor: characterization of purified platelet-derived factor V/Va, J Biol Chem. 279, 2383-93.

50. Broze, G. J., Jr. (1995) Tissue factor pathway inhibitor and the current concept of blood coagulation, Blood Coagul Fibrinolysis. 6 Suppl 1, S7-13.

51. Mast, A. E. \& Broze, G. J., Jr. (1996) Physiological concentrations of tissue factor pathway inhibitor do not inhibit prothrombinase, Blood. 87, 1845-50.

52. Ryan, E. A., Mockros, L. F., Stern, A. M. \& Lorand, L. (1999) Influence of a natural and a synthetic inhibitor of factor XIIla on fibrin clot rheology, Biophys J. 77, 2827-36.

53. Ryan, E. A., Mockros, L. F., Weisel, J. W. \& Lorand, L. (1999) Structural origins of fibrin clot rheology, Biophys J. 77, 2813-26.

54. Fraser, S. R., Booth, N. A. \& Mutch, N. J. (2011) The antifibrinolytic function of factor XIII is exclusively expressed through alpha(2)-antiplasmin cross-linking, Blood. 117, 6371-4.

55. Smith, S. A. \& Morrissey, J. H. (2008) Polyphosphate enhances fibrin clot structure, Blood. 112, 2810-6.

56. Bajzar, L., Manuel, R. \& Nesheim, M. E. (1995) Purification and characterization of TAFI, a thrombin-activable fibrinolysis inhibitor, J Biol Chem. 270, 14477-84.

57. Mao, S. S., Cooper, C. M., Wood, T., Shafer, J. A. \& Gardell, S. J. (1999) Characterization of plasmin-mediated activation of plasma procarboxypeptidase B. Modulation by glycosaminoglycans, $J$ Biol Chem. 274, 35046-52.

58. Konings, J., Govers-Riemslag, J. W., Philippou, H., Mutch, N. J., Borissoff, J. I., Allan, P., Mohan, S., Tans, G., Ten Cate, H. \& Ariens, R. A. (2011) Factor XIla regulates the structure of the fibrin clot independently of thrombin generation through direct interaction with fibrin, Blood. 118, 3942-51. 


\section{Legends}

Figure 1: PolyP enhances coagulation and delays fibrinolysis in human plasma. (A) Combined clotting/fibrinolysis assays were performed in human plasma by adding $75 \mu \mathrm{M}$ poly $\mathrm{P}_{75} 3 \mathrm{~min}$ before recalcifying with $\mathrm{Ca}^{2+}$ in the presence of uPA. Clot formation was monitored as an increase in absorbance over time and subsequent clot lysis as a decrease. PolyP dramatically accelerated the clot formation time in human plasma from around $25 \mathrm{~min}$ to $<5 \mathrm{~min}$. Time to $50 \%$ clot lysis was delayed from $40 \mathrm{~min}$ to $80 \mathrm{~min}$ in the presence of polyP. (B) Clotting assays were performed by preincubating plasma for 2 min with 0 or $75 \mu \mathrm{M}$ polyP 75 before recalcification with $\mathrm{Ca}^{2+}$. The first two bars on the left show that addition of polyP to pooled normal plasma (PNP) dramatically shortened the clotting time, whereas the next two bars show that addition of polyP to factor XII-deficient plasma (XII) did not. The two bars on the right show mixing experiments with normal or factor XIIdeficient plasma in which poly $\mathrm{P}_{75}(75 \mu \mathrm{M})$ was first preincubated for 2 min with one plasma before addition of an equal amount of the other plasma, followed immediately by the addition of $\mathrm{Ca}^{2+}$ to initiate clotting. PolyP dramatically shortened the clotting time only when preincubated with normal plasma, not with factor XII-deficient plasma, even though the final plasma mixtures were identical [8].

Figure 2: PolyP intercepts in the coagulation and fibrinolytic pathways at several points. Coagulation can be initated by two pathways; the extrinsic pathway comprised of tissue factor (TF) and factor VII (VII) or the contact pathway via reciprocal activation of factor XII (XII) and prekallikrein (PK) which associate with negatively charged surfaces via high molecular weight kininogen (HK). Generation of factor XIla (XIla) cleaves downstream factor XI (XI) to factor Xla (Xla) which feed into the intrinsic pathway comprised of factor VIII (VIII) and factor IX (IX). Both pathways converge at the common pathway composed of the prothrombinase complex of factor $\mathrm{Va}(\mathrm{Va})$, factor $\mathrm{Xa}(\mathrm{Xa})$ and prothrombin (PT) ultimately generating thrombin. Thrombin cleaves fibrinogen to fibrin and and activates the transglutaminase factor XIIla (XIIla) generating a cross-linked clot. Cross-linked fibrin is degraded to D-dimer (which can be cleared from the circulation) via the fibrinolytic cascade in which plasmin is generated from plasminogen by tissue plasminogen activator (tPA) or urokinase (UPA). The system is under the control of several inhibitors of which only two are shown; tissue factor pathway inhibitor (TFPI) and activated thrombin activatable fibrinolysis inhibitor (TAFla). PolyP enhances coagulation at several points in the cascade (shown in orange) including; 1 . Stimulating activation of the contact pathway $[7,8,13,36] 2$. Amplifying thrombin-mediated activation of factor $\mathrm{XI}[10]$ and 3. Augmenting conversion of the procofactor factor $\mathrm{V}$ to factor $\mathrm{Va}[8,36]$. By enhancing factor Va generation polyP indirectly interferes with the function of TFPI. The effect of polyP on thrombin generation has down-stream repercussions in terms of fibrinolysis, where it augments activation of TAFI thereby down-regulating fibrinolysis. PolyP (shown in orange) also binds to a number of the haemostatic proteins including thrombin [11], kallikrein [12], XI [10] and XII [7, 13] and fibrin(ogen).

Figure 3: PolyP alters fibrin ultrastructure and down-regulates binding of fibrinolytic proteins to fibrin. (A) Fibrin clots were prepared by incubating fibrinogen with thrombin and $\mathrm{Ca}^{2+}$ in the absence and the presence of polyP 65 . Fibrin clots analysed by confocal microscopy (top panel) and by scanning electron microscopy (bottom panel). Fibrin formed in the presence of polyP is very heterogeneous and is composed of knotted regions interspersed by large pores. (B) Fibrinogen was captured on the surface of a Biacore chip and converted to fibrin by thrombin in the absence and presence of poly $\mathrm{P}_{65}$. Top panel: binding of $125 \mathrm{nM}$ (black), $250 \mathrm{nM}$ (orange), $500 \mathrm{nM}$ (blue), and 1000 $\mathrm{nM}$ (green) plasminogen was analysed (dashed lines). The surface was then treated with plasmin and binding of plasminogen, at the concentrations described above, was repeated (solid lines). Bottom panel: As described for top panel with $31.25 \mathrm{nM}$ (black), $62.5 \mathrm{nM}$ (orange), $125 \mathrm{nM}$ (blue), and 250nM 
(green) tPA instead of plasminogen. PolyP down-regulated binding of plasminogen and tPA especially after plasmin treatment of fibrin. [14] 
Figure 1
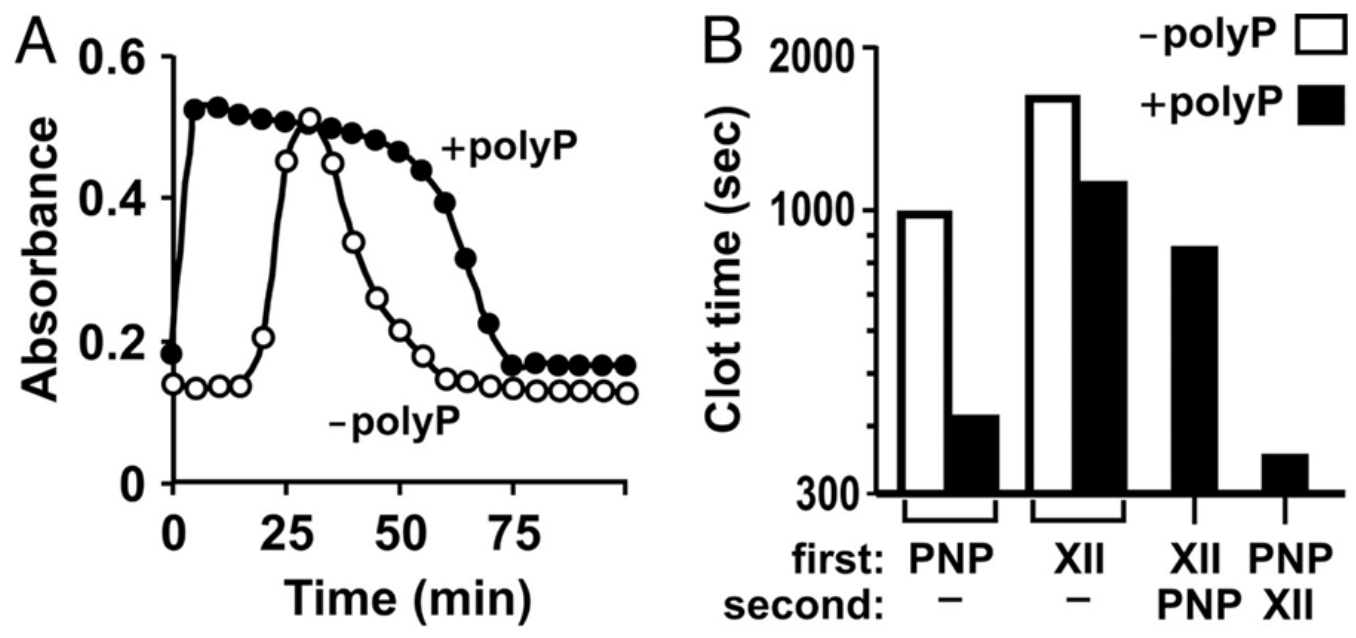
Figure 2

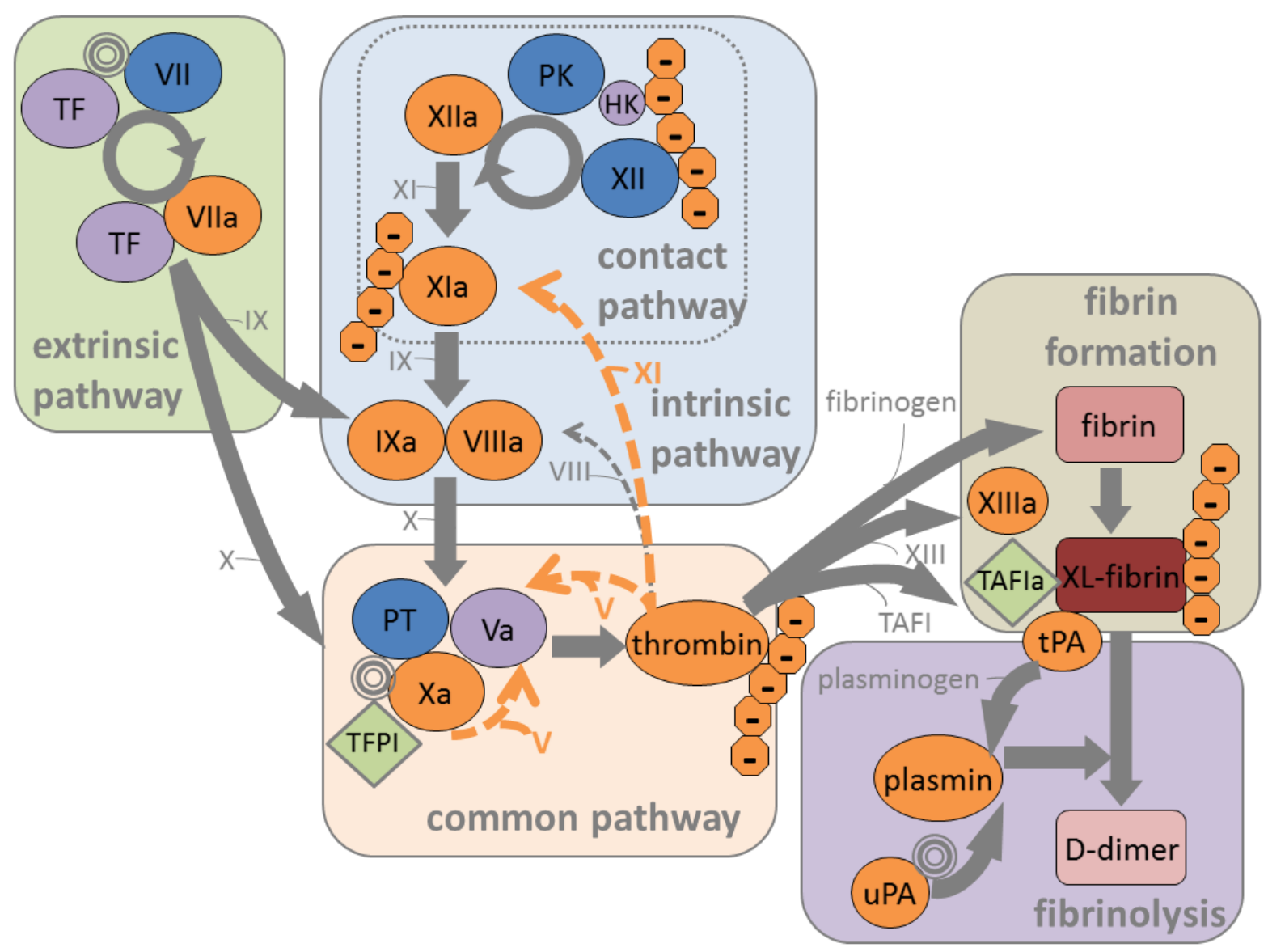


Figure 3
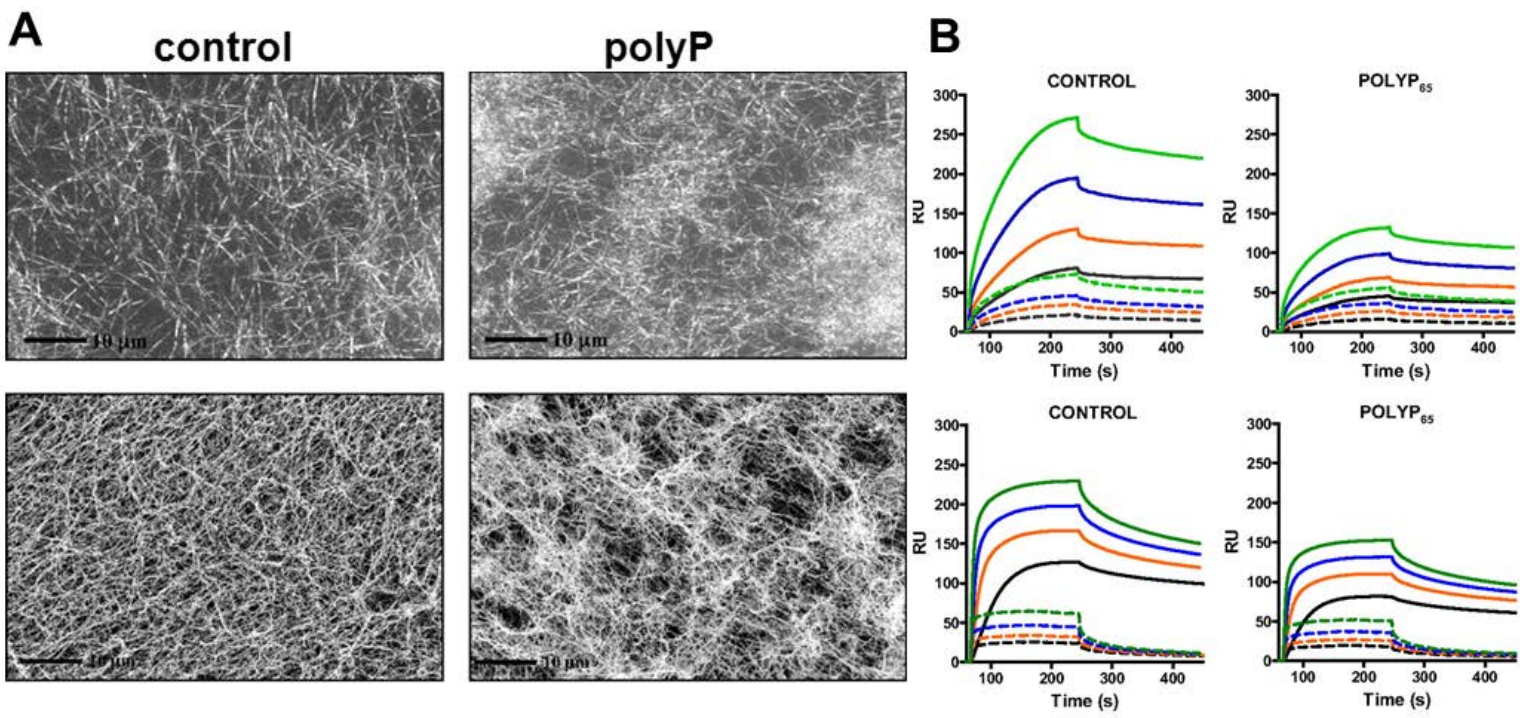